\title{
September 2015 Arizona Thoracic Society Notes
}

The September 2015 Arizona Thoracic Society meeting was held on Wednesday, September 23, 2015 at the Scottsdale Shea Hospital beginning at 6:00 PM. This was a dinner meeting with case presentations. There were 13 in attendance representing the pulmonary, critical care, sleep, and radiology communities.

There were 6 case presentations:

1. Dr. Gerald Schwartzberg presented a case of a 58-year-old woman with a history of Mycobacterium avium presented with cough and malaise. CT revealed a history of lower love centrilobular nodules and scattered ground glass opacities and some bronchiectasis. Sputum revealed Aspergillus fumigatus. IgE was normal but IgA was deficient at $20 \mathrm{mg} / \mathrm{dl}$ (normal $80-350 \mathrm{mg} / \mathrm{dl}$ ). She was started on itraconazole and clinically improved. Many questioned whether the Aspergillus was the cause of her pneumonia and some questioned the association of the IgA deficiency with her overall clinical picture.

2. Dr. Schwartzberg presented a second case of a 92-year-old former opera singer who had a past diagnosis of asthma but without airflow obstruction, gastroesophageal reflux disease, and myelodysplastic syndrome. CT scan revealed mosaic areas most consistent with hypoperfusion secondary to air trapping. Complete pulmonary function testing revealed only a markedly decreased DLco. She had oxygen desaturation with exercise. Clinically she did not respond to a bronchodilator. Most were perplexed as the cause of her overall clinical picture.

3. Dr. Schwartzberg presented a third case of a morbidly obese 61-year-old woman who presented with shortness of breath. CT scan showed some scattered lung nodules in her lower lobes. Laboratory evaluation including cocci serologies were negative. A needle biopsy of one of the lung nodules was nondiagnostic and she was empirically begun on fluconazole. She clinically improved. Many thought this could be possibly Valley fever and she should be followed.

4. Dr. Alan Thomas presented a 66-year-old man with a history of lymphoma about 10 years earlier who presented with some enlarging lymph nodes. Thoracic CT scan was performed as part of his evaluation and showed some areas of emphysema with scattered ground glass opacities. It was felt the radiologic pattern was most consistent with respiratory bronchiolitis with fibrosis (2).

5. Dr. Thomas also presented a case of an 82-year-old former smoker who quit about a year ago who presented with weight loss and minimal cough. Thoracic CT scan showed a large pleural mass with pleural effusion surrounding the right lung as well as pleural plaques. He did have a history of asbestos exposure in the Navy. Thoracentesis showed a nondiagnostic exudative effusion. A biopsy was performed which was consistent with a large cell neuroendocrine tumor.

6. Dr. Lewis Wesselius presented a 65-year-old man with exertional dyspnea and possible interstitial lung disease. He has a history of a Ross procedure (replacement of a bicuspid aortic valve with the pulmonic valve) and obstructive sleep apnea. Chest $\mathrm{x}$-ray was unremarkable. Complete pulmonary function testing was normal. Thoracic CT scan showed peripheral reticulations especially in the lower lobes. A 
video-assisted thorascopic biopsy (VATS) was performed. Histology showed scattered fibroblast foci with scattered fibrosis with airway centricity. It was unclear whether this was usual interstitial fibrosis or chronic hypersensitivity pneumonitis. He was started on prednisone because his picture was felt to be most consistent with chronic hypersensitivity pneumonitis (1). Unfortunately, chronic hypersensitivity pneumonitis with features of UIP appears to carry a worse prognosis.

There being no further business, the meeting was adjourned about 7:30 PM. The next meeting will be in Phoenix at Scottsdale Shea on Wednesday, November 18 at 6:30 PM.

Richard A. Robbins, MD

Editor, SWJPCC

\section{References}

1. Reddy TL, Mayo J, Churg A. Respiratory bronchiolitis with fibrosis. High-resolution computed tomography findings and correlation with pathology. Ann Am Thorac Soc. 2013;10(6):590-601. [CrossRef] [PubMed]

2. Myers JL. Hypersensitivity pneumonia: the role of lung biopsy in diagnosis and management. Mod Pathol. 2012;25 Suppl 1:S58-67. [CrossRef] [PubMed] 